arXiv: hep-th/0412255

Report IJS/TP-25/04

\title{
Kaluza-Klein Theory without Extra Dimensions: Curved Clifford Space
}

\author{
Matej Pavšič \\ Jožef Stefan Institute, Jamova 39, 1000 Ljubljana, Slovenia \\ e-mail: matej.pavsic@ijs.si
}

\begin{abstract}
A theory in which 16-dimensional curved Clifford space ( $C$-space) provides a realization of Kaluza-Klein theory is investigated. No extra dimensions of spacetime are needed: "extra dimensions" are in $C$-space. It is shown that the covariant Dirac equation in $C$-space contains Yang-Mills fields of the $\mathrm{U}(1) \times \mathrm{SU}(2) \times \mathrm{SU}(3)$ group as parts of the generalized spin connection of the $C$-space.
\end{abstract}

\section{Introduction}

There is more to spacetime than usually envisaged in special or general relativity. Even at the classical level, besides the bosonic coordinates it is customary to include Grassmann odd coordinates into the game (see, e.g. [1]). They provide a description of spinning degrees of freedom. An alternative way [2]-[7] of extending spacetime is to consider the corresponding Clifford space (shortly $C$-space) generated by basis vectors $\gamma_{\mu}$. A point of $C$ space is described by a set of multivector coordinates $\left(s, x^{\mu}, x^{\mu \nu}, \ldots\right)$ which altogether with the corresponding basis elements $\left(1, \gamma_{\mu}, \gamma_{\mu \nu}, \ldots\right)$ form a Clifford aggregate or polyvector $X$. It is well known [8, 9] that the elements of the right or left minimal ideals of Clifford algebra

\footnotetext{
${ }^{0 \dagger} \mathrm{A}$ revised version of this paper will appear in Physics Letters B.
} 
can be used to represent spinors. Therefore, a coordinate polyvector $X$ automatically contains spinor as well as bosonic coordinates. In refs. [10, 11] it was proposed to formulate string theory in terms of polyvectors, and thus avoid using a higher dimensional spacetime. Spacetime can be 4-dimensional, whilst the extra degrees of freedom ("extra dimensions") necessary for consistency of string theory are in Clifford space.

In this paper we propose to go even further: 16-dimensional curved Clifford space can provide a realization of the Kaluza-Klein idea [10]. We do not need to assume that spacetime has more than four dimensions. The "extra dimensions" are in Clifford space. This approach has seeds in refs. [12, 13, but explicitly it was formulated in refs. [10, 14]. We will first investigate some basic aspects of the classical general relativity-like theory in $C$-space. Then we pass to quantum theory and rewrite the Dirac-like equation in curved $C$-space and show that the corresponding generalized spin connection contains Yang-Mills fields describing fundamental interactions.

Although other authors in a number of very illuminating and penetrating papers [15] have investigated unified models of fundamental interactions within the framework of Clifford algebra, they have not fully employed the concept of Clifford space, together with the $C$-space metric, affine and spin connection, polyvector-valued wave function [6], which all enable to formulate a Kaluza-Klein like theory in 16-dimensional Clifford space defined over 4-dimensional spacetime. As far as I know this is a novel approach (see also refs. 12, 13, 10, 14]).

\section{Clifford space as a generalization of spacetime}

Since pioneering works by Hestenes [16], Clifford algebra has been extensively investigated (see e.g. refs. [17]-22]). Some researchers [2]-[7] proposed to replace spacetime with a larger geometric structure which is based on Clifford algebra. This has led to the concept of Clifford space (shortly $C$-space).

Suppose we have an $n$-dimensional space $V_{n}$, not necessarily flat. At every point $x \in V_{n}$ we have a flat tangent space, its basis being given in terms of $n$ orthonormal vectors $\gamma_{a}$, $a=1,2, \ldots, n$ satisfying the Clifford algebra relations

$$
\gamma_{a} \cdot \gamma_{b} \equiv \frac{1}{2}\left(\gamma_{a} \gamma_{b}+\gamma_{b} \gamma_{a}\right)=\eta_{a b} \mathbf{1}
$$


where $\eta_{a b}$ is a pseudo-Euclidean metric whose signature is kept arbitrary at this stage. The basis vectors $\gamma_{a}$ form a local basis in $V_{n}$ and they generate the Clifford algebra $\mathcal{C}_{M_{n}}$. The basis of the latter algebra is given by the set

$$
\left\{\gamma_{A}\right\}=\left\{\mathbf{1}, \gamma_{a_{1}}, \gamma_{a_{1} a_{2}}, \ldots, \gamma_{a_{1} a_{2} \ldots a_{n}}\right\}, \quad a_{1}<a_{2}<\ldots<a_{r}, \quad r=1,2, \ldots, n
$$

where $\gamma_{a_{1} a_{2} \ldots a_{r}} \equiv \gamma_{a_{1}} \wedge \gamma_{a_{2}} \wedge \ldots \wedge \gamma_{a_{r}} \equiv \frac{1}{r !}\left[\gamma_{a_{1}}, \gamma_{a_{2}}, \ldots, \gamma_{a_{r}}\right]$ is the wedge product.

From a local basis $\left\{\gamma_{a}\right\}$ we can switch to a coordinate basis $\left\{\gamma_{\mu}\right\}$ according to the relation

$$
\gamma_{\mu}=e_{\mu}^{a} \gamma_{a}
$$

where $e_{\mu}^{a}=\gamma_{\mu} \cdot \gamma^{a}$ is the vielbein field.

The coordinate basis vectors satisfy

$$
\gamma_{\mu} \cdot \gamma_{\nu} \equiv \frac{1}{2}\left(\gamma_{\mu} \gamma_{\nu}+\gamma_{\nu} \gamma_{\nu}\right)=g_{\mu \nu}
$$

where $g_{\mu \nu}$ is the metric of $V_{n}$. We may use $\gamma_{\mu}$ as generators of Clifford algebra with the basis

$$
\left\{\gamma_{M}\right\}=\left\{\gamma, \gamma_{\mu_{1}}, \gamma_{\mu_{1} \mu_{2}}, \ldots, \gamma_{\mu_{1} \ldots \mu_{n}}\right\}, \quad \mu_{1}<\mu_{2}<\ldots<\mu_{r}, \quad r=1,2, \ldots, n
$$

where $\gamma=\mathbf{1}$ and $\gamma_{\mu_{1} \ldots \mu_{r}} \equiv \gamma_{\mu_{1}} \wedge \gamma_{\mu_{2}} \wedge \ldots \wedge \gamma_{\mu_{r}}$. Since $\gamma_{\mu}$ and $g_{\mu \nu}$ depend on position, we have different Clifford algebras $\mathcal{C}_{V_{n}}$ at different points $x \in V_{n}$. The continuous set of all those algebras over a domain of $V_{n}$ forms a manifold $\mathcal{C}_{V_{n}}(x)$ which is usually called Clifford bundle or Clifford manifold.

In this paper we propose to introduce a more general Clifford manifold (see also [3, 6, 12]). Let us start from the flat Clifford space with basis (2). We then perform transition to a curved Clifford space with basis $\left\{\gamma_{M}\right\}$ by means of the relation

$$
\gamma_{M}=e_{M}^{A} \gamma_{A}
$$

where $e_{M}{ }^{A}$ is the fielbein field in $C$-space. The latter relation is more general than (3). Explicitly it reads

$$
\begin{aligned}
& \gamma=e_{\mathbf{o}} \underline{\mathbf{o}} \mathbf{1}+e_{\mathbf{o}}^{a_{1}} \gamma_{a_{1}}+e_{\mathbf{o}}^{{ }^{a} a_{2}} \gamma_{a_{1} a_{2}}+\ldots+e_{\mathbf{o}}{ }^{a_{1} \ldots a_{n}} \gamma_{a_{1} \ldots a_{n}} \\
& \gamma_{\mu_{1}}=e_{\mu_{1}} \underline{\mathbf{o}} \mathbf{1}+e_{\mu_{1}}^{a_{1}} \gamma_{a_{1}}+e_{\mu_{1}}{ }^{a_{1} a_{2}} \gamma_{a_{1} a_{2}}+\ldots+e_{\mu_{1}}{ }^{a_{1} \ldots a_{n}} \gamma_{a_{1} \ldots a_{n}} \\
& \gamma_{\mu_{1} \mu_{2}}=e_{\mu_{1} \mu_{2}} \underline{\mathbf{o}} \mathbf{1}+e_{\mu_{1} \mu_{2}}{ }^{a_{1}} \gamma_{a_{1}}+e_{\mu_{1} \mu_{2}}{ }^{a_{1} a_{2}} \gamma_{a_{1} a_{2}}+\ldots+e_{\mu_{1} \mu_{2}}{ }^{a_{1} \ldots a_{n}} \gamma_{a_{1} \ldots a_{n}} \\
& \vdots \\
& \gamma_{\mu_{1} \ldots \mu_{n}}=e_{\mu_{1} \ldots \mu_{n}} \underline{\mathbf{o}} \mathbf{1}+e_{\mu_{1} \ldots \mu_{n}}{ }^{a_{1}} \gamma_{a_{1}}+e_{\mu_{1} \ldots \mu_{n}}{ }^{a_{1} a_{2}} \gamma_{a_{1} a_{2}}+\ldots+e_{\mu_{1} \ldots \mu_{n}}{ }^{a_{1} \ldots a_{n}} \gamma_{a_{1} \ldots a_{n}}
\end{aligned}
$$


where $\gamma_{a_{1} \ldots a_{r}} \equiv \gamma_{a_{1}} \wedge \ldots \wedge \gamma_{a_{r}}$, whilst $\gamma_{\mu_{1} \ldots \mu_{r}}$ are no longer defined as the wedge product.

From the basis elements $\gamma_{M}$ we can define the metric of $C$-space according to

$$
G_{M N}=\gamma_{M}^{\ddagger} * \gamma_{N}
$$

Here ' $f$ ' denotes the reversion, that is the operation which reverses the order of the gener-

ators $\gamma_{a}$ (for example, $\gamma_{a_{1} a_{2} a_{3}}^{\ddagger}=\gamma_{a_{3} a_{2} a_{1}}$ ), whilst '*' denotes the scalar product between two Clifford numbers $A$ and $B$

$$
A * B=\langle A B\rangle_{0}
$$

The quantities $\gamma_{M}, e_{M}^{A}, G_{M N}$ are now assumed to depend on position in $C$-space which can be parametrized by $C$-space coordinates vector fields

$$
X=x^{M} \gamma_{M}=s \gamma+x^{\mu_{1}} \gamma_{\mu_{1}}+x^{\mu_{1} \mu_{2}} \gamma_{\mu_{1} \mu_{2}}+\ldots+x^{\mu_{1} \ldots \mu_{n}} \gamma_{\mu_{1} \ldots \mu_{n}}
$$

In $C$-space the multivector grade is relative to a chosen basis, and a coordinates transformation in $C$-space in general changes the grade of $\gamma_{\mu_{1} \ldots \mu_{r}}$. Thus even if an object appears as a 1-vector with respect to a coordinate basis $\gamma_{\mu}$, it is a polyvector (a superposition of multivectors) with respect to the local basis $\gamma_{a}$.

We have thus a curved Clifford space ( $C$-space). A point of $C$-space is described by coordinates $x^{M}$. A coordinate basis is $\left\{\gamma_{M}\right\}$, whilst a local (flat) basis is $\left\{\gamma_{A}\right\}$. The tetrad field is given by the scalar product $e_{M}^{A}=\gamma_{M}^{\ddagger} * \gamma^{A}$.

The multivector coordinates $s, x^{\mu_{1}}, x^{\mu_{1} \mu_{2}}, \ldots, x^{\mu_{1} \ldots \mu_{n}}$ provide a description of oriented $r$-dimensional areas. In refs. [7] a physical interpretation was given, namely that the multivector coordinates can be used to describe extended objects, such as closed branes.

\section{On the realization of Kaluza-Klein theory in curved Clifford space}

The basic idea of Kaluza-Klein theory is that spacetime has more than four dimensions. The extra dimensions of curved spacetime manifest as gauge fields describing the fundamental interactions. Instead of introducing extra dimensions, we can investigate a theory which starts from 4-dimensional spacetime and then generalize it to curved Clifford space.

Let us first consider the equation of geodesic in curved $C$-space. We can envisage that physical objects are described in terms of $x^{M}=\left(s, x^{\mu}, x^{\mu \nu}, \ldots\right)$. The first straightforward possibility is to introduce a single parameter $\tau$ and consider a mapping $\tau \rightarrow x^{M}=X^{M}(\tau)$ 
where $X^{M}(\tau)$ are 16 embedding functions that describe a worldline in $C$-space. From the point of view of $C$-space, $X^{M}(\tau)$ describe a worldline of a "point particle": at every value of $\tau$ we have a point in $C$-space. But from the perspective of the underlying 4-dimensional spacetime, $X^{M}(\tau)$ describe an extended object, sampled by the center of mass coordinates $X^{\mu}(\tau)$ and the coordinates $X^{\mu_{1} \mu_{2}}(\tau), \ldots, X^{\mu_{1} \mu_{2} \mu_{3} \mu_{4}}(\tau)$. They are a generalization of the center of mass coordinates in the sense that they provide information about the object's 2 -vector, 3 -vector, and 4-vector extension and orientation ${ }^{1}$.

The dynamics of such an object is determined by the action

$$
\begin{aligned}
& I[X]=\int \mathrm{d} \tau\left(\dot{X}^{M} \dot{X}^{N} G_{M N}\right)^{1 / 2} \\
& \delta X^{M}: \frac{1}{\sqrt{\dot{X}^{2}}} \frac{\mathrm{d}}{\mathrm{d} \tau}\left(\frac{\dot{X}^{M}}{\sqrt{\dot{X}^{2}}}\right)+\Gamma_{J K}^{M} \frac{\dot{X}^{J} \dot{X}^{K}}{\dot{X}^{2}}=0
\end{aligned}
$$

Here $\dot{X}^{J} \equiv \mathrm{d} X^{J} / \mathrm{d} \tau$ is the derivative with respect to an arbitrary monotonically increasing parameter $\tau$, and $\Gamma_{J K}^{M}$ is the connection defined according to ${ }^{2}$

$$
\partial_{M} \gamma_{N}=\Gamma_{M N}^{K} \gamma_{K}
$$

The above relation is a generalization [12] of the well known relation [16].

When the derivative $\partial_{M}$ acts on a polyvector $A=A^{N} \gamma_{N}$ we obtain the covariant derivative $\mathrm{D}_{M}$ acting on the components $A^{N}$ :

$$
\partial_{M}\left(A^{N} \gamma_{N}\right)=\partial_{M} A^{N} \gamma_{N}+A^{N} \partial_{M} \gamma_{N}=\left(\partial_{M} A^{N}+\Gamma_{M K}^{N} A^{K}\right) \gamma_{N} \equiv \mathrm{D}_{M} A^{N} \gamma_{N}
$$

Here the $A^{N}$ are scalar components of $A$, and $\partial_{M} A^{N}$ is just the ordinary partial derivative with respect to $X^{M}$ :

$$
\partial_{M} \equiv\left(\frac{\partial}{\partial s}, \frac{\partial}{\partial x^{\mu_{1}}}, \frac{\partial}{\partial x^{\mu_{1} \mu_{2}}}, \frac{\partial}{\partial x^{\mu_{1} \ldots \mu_{n}}}\right)
$$

The derivative $\partial_{M}$ behaves as a partial derivative when acting on scalars, and it defines a connection when acting on a basis $\left\{\gamma_{M}\right\}$. It has turned out very practical ${ }^{3}$ to use the easily writable symbol $\partial_{M}$ which — when acting on a polyvector - cannot be confused with partial derivative.

When inspected from the 4-dimensional spacetime, the equation of geodesic (12) contains besides the usual gravitation also other interactions. They are encoded in the metric

\footnotetext{
${ }^{1} \mathrm{~A}$ systematic and detailed treatment is in ref. [7.

${ }^{2}$ For more details see refs. [16, 6, 12

${ }^{3}$ Especially when doing long calculation (which is usually the job of a theoretical physicist) it is much easier and quicker to write $\partial_{M}$ than $\square_{M}, \nabla_{M}, D_{\gamma_{M}}, \nabla_{\gamma_{M}}$ which all are symbols used in the literature.
} 
components $G_{M N}$ of $C$-space. Gravity is related to the components $G_{\mu \nu}, \mu, \nu=0,1,2,3$, while gauge fields are related to the components $G_{\mu \bar{M}}$, where the index $\bar{M} \neq \nu$ assumes 12 possible values, excluding the four values $\nu=0,1,2,3$. In addition, there are also interactions due to the components $G_{\bar{M} \bar{N}}$, but they have not the property of the ordinary Yang-Mills fields.

If we now consider the known fundamental interactions of the standard model we see that besides gravity we have 1 photon described by the abelian gauge field $A_{\mu}, 3$ weak gauge bosons described by gauge fields $W_{\mu}^{a}, a=1,2,3$, and 8 gluons described $A_{\mu}^{c}, c=1,2, \ldots, 8$. Altogether there are 12 gauge fields.

Interestingly, the number of mixed components $G_{\mu \bar{M}}=\left(G_{\mu[\mathbf{o}]}, G_{\mu[\alpha \beta]}, G_{\mu[\alpha \beta \rho]}, G_{\mu[\alpha \beta \rho \sigma]}\right)$ of the $C$-space metric tensor $G_{M N}$ coincides with the number of gauge fields in the standard model ${ }^{4}$. For fixed $\mu$, there are 12 mixed components of $G_{\mu \bar{M}}$ and 12 gauge fields $A_{\mu}, W_{\mu}^{a}, A_{\mu}^{c}$. This coincidence is fascinating and it may indicate that the known interactions are incorporated in curved Clifford space.

Good features of $C$-space are the following:

(i) We do not need to introduce extra dimensions of spacetime. We stay with 4dimensional spacetime $V_{4}$, and yet we can proceed à la Kaluza-Klein. The extra degrees of freedom are in $C$-space, generated by a basis of $V_{4}$.

(ii) We do not need to compactify the extra "dimensions". The extra dimensions of $C$ space, namely $s, x^{\mu \nu}, x^{\mu \nu \rho}, x^{\mu \nu \rho \sigma}$ are not just like the ordinary dimensions of spacetime considered in the usual Kaluza-Klein theories. The coordinates $x^{\mu \nu}, x^{\mu \nu \rho}, x^{\mu \nu \rho \sigma}$ are related to oriented $r$-surfaces, $r=2,3$, 4 , by which we sample extended objects. Those degrees of freedom are in principle not hidden from our direct observation, therefore we do not need to compactify such "internal" space.

(iii) The number of the mixed metric components $G_{\mu \bar{M}}$ (for fixed $\mu$ ) is 12 , precisely the same as the number of gauge fields in the standard model.

\footnotetext{
${ }^{4}$ The numbers of the independent indices $[\mathbf{o}],[\alpha \beta],[\alpha \beta \rho],[\alpha \beta \rho \sigma]$ are respectively $1,6,4,1$ which sums to 12 .
} 


\section{The Dirac equation in curved $C$-space}

\subsection{Spinors as members of left ideals}

How precisely the curved $C$-space is related to Yang-Mills gauge fields can be demonstrated by considering a generalization of the Dirac equation to curved $C$-space.

Let $\Phi(X)$ be a polyvector valued field over coordinates polyvector field $X=x^{M} \gamma_{M}$ :

$$
\Phi=\phi^{A} \gamma_{A}
$$

where $\gamma_{A}, A=1,2, \ldots, 16$, is a local (flat) basis of $C$-space (see eq.(2)) and $\phi^{A}$ the projections (components) of $\Phi$ onto the basis $\left\{\gamma_{A}\right\}$. We will suppose that in general $\phi^{A}$ are complex-valued scalar quantities.

Instead of the basis $\left\{\gamma_{A}\right\}$ one can consider another basis, which is obtained after multiplying $\gamma_{A}$ by 4 independent primitive idempotents $[9$

$$
P_{i}=\frac{1}{4}\left(\mathbf{1}+a_{i} \gamma_{A}+b_{i} \gamma_{B}+c_{i} \gamma_{C}\right), \quad i=1,2,3,4
$$

such that

$$
P_{i}=\frac{1}{4}\left(\mathbf{1}+a_{i} \gamma_{A}\right)\left(\mathbf{1}+b_{i} \gamma_{B}\right), \quad \gamma_{A} \gamma_{B}=\gamma_{C}, \quad c_{i}=a_{i} b_{i}
$$

Here $a_{i}, b_{i}, c_{i}$ are complex numbers chosen so that $P_{i}^{2}=P_{i}$. For explicit and systematic construction see [9], 23].

By means of $P_{i}$ we can form minimal ideals of Clifford algebra. A basis of left (right) minimal ideal is obtained by taking one of $P_{i}$ and multiply it from the left (right) with all 16 elements $\gamma_{A}$ of the algebra:

$$
\gamma_{A} P_{i} \in \mathcal{I}_{i}^{L}, \quad P_{i} \gamma_{A} \in \mathcal{I}_{i}^{R}
$$

Here $\mathcal{I}_{i}^{L}$ and $\mathcal{I}_{i}^{R}, i=1,2,3,4$ are four independent minimal left and right ideals, respectively. For a fixed $i$ there are 16 elements $P_{i} \gamma_{A}$, but only 4 amongst them are different, the remaining elements are just repetition of those 4 different elements.

Let us denote those different elements $\xi_{\alpha i}, \alpha=1,2,3,4$. They form a basis of the $i$-th left ideal. Every Clifford number can be expanded either in terms of $\gamma_{A}=$ $\left(\mathbf{1}, \gamma_{a_{1}}, \gamma_{a_{1} a_{2}}, \gamma_{a_{1} a_{2} a_{3}}, \gamma_{a_{1} a_{2} a_{3} a_{4}}\right)$ or in terms of $\xi_{\alpha i}=\left(\xi_{\alpha 1}, \xi_{\alpha 2}, \xi_{\alpha 3}, \xi_{\alpha 4}\right)$ :

$$
\Phi=\phi^{A} \gamma_{A}=\Psi=\psi^{\alpha i} \xi_{\alpha i}=\psi^{\tilde{A}} \xi_{\tilde{A}}
$$


In the last step we introduced a single spinor index $\tilde{A}$ which runs over all 16 basis elements that span 4 independent left minimal ideals. Explicitly, eq. (20) reads

$$
\Psi=\psi^{\tilde{A}} \xi_{\tilde{A}}=\psi^{\alpha 1} \xi_{\alpha 1}+\psi^{\alpha 2} \xi_{\alpha 2}+\psi^{\alpha 3} \xi_{\alpha 3}+\psi^{\alpha 4} \xi_{\alpha 4}
$$

Eq.(20) or (21) represents a direct sum of four independent 4-component spinors, each living in a different left ideal $\mathcal{I}_{i}^{L}$.

In ref. [6] it was proposed ${ }^{5}$ that the polyvector valued wave function satisfies the Dirac equation in $C$-space:

$$
\partial \Psi \equiv \gamma^{M} \partial_{M} \Psi=0
$$

The derivative $\partial_{M}$ is the same derivative introduced in eqs. (13) and (14). Now it acts on the object $\Psi$ which is expanded in terms of the 16 basis elements $\xi_{\tilde{A}}$, which in turn can be written as a superposition of basis elements $\gamma_{A}$ of Clifford algebra. The action of $\partial_{M}$ on the spinor basis elements $\xi_{\tilde{A}}$ gives the spin connection:

$$
\partial_{M} \xi_{\tilde{A}}=\Gamma_{M}^{\tilde{B}}{ }_{\tilde{A}} \xi_{\tilde{B}}
$$

Using the expansion (21) and eq. (23) we find

$$
\gamma^{M} \partial_{M}\left(\psi^{\tilde{A}} \xi_{\tilde{A}}\right)=\gamma^{M}\left(\partial_{M} \psi^{\tilde{A}}+\Gamma_{M}^{\tilde{A}}{ }_{\tilde{B}} \psi^{\tilde{B}}\right) \xi_{\tilde{A}}=0
$$

This is just a generalization of the ordinary Dirac equation in curved spacetime. Instead of curved spacetime, spin connection and the Dirac spinor, we have now curved Clifford space, generalized spin connection and the generalized spinor $\psi^{\tilde{A}}$ which incorporates 4 independent Dirac spinors, as indicated in eq. (21).

We may now use the relations

$$
\xi^{\tilde{A}^{\ddagger}} * \xi_{\tilde{B}} \equiv\left\langle\xi^{\tilde{A}^{\ddagger}} \xi_{\tilde{B}}\right\rangle_{S}=\delta_{\tilde{B}}^{\tilde{A}}
$$

and

$$
\left\langle\xi^{\tilde{C}^{\ddagger}} \gamma^{M} \xi_{\tilde{A}}\right\rangle_{S}=\left(\gamma^{M}\right)_{\tilde{A}}^{\tilde{C}}
$$

where the operation \langle\rangle$_{S} \equiv \operatorname{Tr}\langle\rangle_{0}$ takes the scalar part of the expression and then performs the trace. We normalize $\xi_{\tilde{A}}$ so that (25) is fulfilled. By means of (25) we can project eq.(24) onto its component form

$$
\left(\gamma^{M}\right)_{\tilde{A}}^{\tilde{C}}\left(\partial_{M} \psi^{\tilde{A}}+\Gamma_{M}{ }_{\tilde{B}}^{\tilde{B}} \psi^{\tilde{B}}\right)=0
$$

\footnotetext{
${ }^{5}$ See also illuminating works in refs. [3, 24].
} 
The spinor indices $\tilde{A}, \tilde{B}$ can be omitted and eq. (27) written simply as

$$
\gamma^{M}\left(\partial_{M}+\Gamma_{M}\right) \psi=0
$$

We see that in the geometric form of the Dirac equation (22) spin connection is automatically present through the action of the derivative $\partial_{M}$ on a polyvector $\Psi$ written as a superposition of basis spinors $\xi_{\tilde{A}}$. The reader has to be careful (i) not to confuse our symbol $\partial_{M}$ (when acting on a polyvector) with a partial derivative, (ii) not to miss the fact that $\Psi$ in eq. (22) is a Clifford algebra valued object, not just a component spinor, and (iii) not hastily think that eq. (22) lacks covariance.

\subsection{Yang-Mills gauge fields as spin connection in $C$-space}

Let us define generators of the transformations (i.e., local rotations in $C$-space) according to

$$
\Sigma_{A B}= \begin{cases}\frac{1}{2}\left[\gamma_{A}, \gamma_{B}\right], & \text { if } A \neq \underline{\mathbf{o}}, B \neq \mathbf{o} \\ \mathbf{1} \gamma_{B}, & \text { if } A=\underline{\mathbf{o}}\end{cases}
$$

We also have $\Sigma_{A B}=f_{A B}{ }^{C} \gamma_{C}$, where $f_{A B}{ }^{C}$ are constants.

A generic transformation in $C$-space which maps a polyvector $\Psi$ into another polyvector $\Psi^{\prime}$ is given by

$$
\Psi^{\prime}=R \Psi S
$$

where

$$
R=\mathrm{e}^{\frac{1}{4} \Sigma_{A B} \alpha^{A B}}=\mathrm{e}^{\gamma_{A} \alpha^{A}} \text { and } S=\mathrm{e}^{\frac{1}{4} \Sigma_{A B} \beta^{A B}}=\mathrm{e}^{\gamma_{A} \beta^{A}}
$$

Here $\alpha^{A B}$ and $\beta^{A B}$, or equivalently $\alpha^{A}=f_{C D}{ }^{A} \alpha^{C D}$ and $\beta^{A}=f_{C D}{ }^{A} \beta^{C D}$, are parameters of the transformation.

In general, eq. (30) allows for the transformation which maps a basis element $\gamma_{A}$ into a mixture of basis elements. In particular, we have the following three interesting cases:

(i) $\alpha^{A B} \neq 0, \beta^{A B}=-\alpha^{A B}$. Then we have

$$
\Psi^{\prime}=R \Psi R^{-1}
$$

This is the transformation which preserves the structure of Clifford algebra, i.e., it maps the basis elements $\gamma_{A}$ into another basia element $\gamma_{A^{\prime}}$ of the same Clifford algebra.

(ii) $\alpha^{A B} \neq 0, \beta^{A B}=0$. Then we have

$$
\Psi^{\prime}=R \Psi
$$


This is the transformation which maps a basis spinor $\xi_{\alpha i}$ into another basis spinor $\xi_{\alpha i}^{\prime}$ belonging to the same left ideal:

$$
\xi_{\alpha i} \subset \mathcal{I}_{i}^{L} \rightarrow \xi_{\alpha i}^{\prime}=R \xi_{\alpha i} \subset \mathcal{I}_{i}^{L}
$$

(iii) $\alpha^{A B}=0, \quad \beta^{A B} \neq 0$. Then

$$
\Psi^{\prime}=\Psi S
$$

This is the transformation that maps right ideal into the right ideals:

$$
\xi_{\alpha i} \subset \mathcal{I}_{i}^{R} \rightarrow \xi_{\alpha i}^{\prime}=\xi_{\alpha i} S \subset \mathcal{I}_{i}^{R}
$$

In general, for the transformation (30) we have

$$
\Psi^{\prime}=\psi^{\tilde{A}} R \xi_{\tilde{A}} S=\psi^{\tilde{A}} U_{\tilde{A}}^{\tilde{B}} \xi_{\tilde{B}}=\psi^{\prime \tilde{A}} \xi_{\tilde{A}}
$$

where

$$
\psi^{\prime \tilde{A}}=U^{\tilde{A}}{ }_{\tilde{B}} \psi^{\tilde{B}}
$$

This transformation, in general, mixes right and left ideals. Eq. (38) can be considered as matrix equation in the space spanned by the generalized spinor indices $\tilde{A}, \tilde{B}$ :

$$
\psi^{\prime}=U \psi
$$

where $U$ is a $16 \times 16$ matrix, whilst $\psi$ and $\psi^{\prime}$ are columns with 16 elements. From (37), (38) it follows that $U=\hat{R} \otimes \hat{S}^{\mathrm{T}}$, where $\hat{R}$ and $\hat{S}$ are $4 \times 4$ matrices representing the Clifford numbers $R$ and $S$. That is, $U$ is the direct product of $\hat{R}$ and the transpose $\hat{S}^{\mathrm{T}}$ of $\hat{S}$, and it belongs, in general, to the group $G L(4, C) \times G L(4, C)$. The group is local, because the basis elements $\gamma_{A}$ entering the definition (32) depend on position $X$ in $C$-space according to the relation analogous to (13), and also the group parameters $\alpha^{A}, \beta^{A}$ in general depend on $X$.

We now require that the $C$-space Dirac equation is invariant under the transformations (301), (39):

$$
\partial^{\prime} \Psi^{\prime}=\partial \Psi=\gamma^{M} \partial_{M}\left(\psi^{\tilde{A}} \xi_{\tilde{A}}\right)
$$

After using eq.(23) we then find ${ }^{6}$

$$
\Gamma_{M \tilde{A}}^{\prime \tilde{B}}=U_{\tilde{D}}^{\tilde{B}} U^{\tilde{C}}{ }_{\tilde{A}} \Gamma_{M \tilde{C}}^{\tilde{D}}+\partial_{M} U^{\tilde{D}} U_{\tilde{D}}^{\tilde{B}}
$$

\footnotetext{
${ }^{6}$ More details will be provided elsewhere.
} 
This is the transformation for the generalized spin connection (i.e., the connection in $C$ space). In matrix notation ${ }^{7}$ this reads

$$
\Gamma_{M}^{\prime}=U \Gamma_{M} U^{-1}+U \partial_{M} U^{-1}
$$

We see that $\Gamma_{M}$ transforms as a non abelian gauge field. The most general gauge group here is ${ }^{8} \mathrm{GL}(4, \mathrm{C}) \times \mathrm{GL}(4, \mathrm{C})$. As subgroups it contains for instance $\mathrm{SL}(2, \mathrm{C})$ and $\mathrm{U}(1) \times$ $\mathrm{SU}(2) \times \mathrm{SU}(3)$. The former group describes the Lorentz transformations in Minkowski space (which is a subspace of $C$-space), whilst the latter group coincides with the gauge group of the standard model which describes electroweak and strong interaction. We have thus demonstrated that the generally covariant Dirac equation in 16-dimensional curved $C$-space contains the coupling of spinor fields $\psi^{\tilde{A}}$ with non abelian gauge fields $\Gamma_{M}{ }^{\tilde{A}}$ which altogether form the spin connection in $C$-space.

Whether this indeed provides a description of the standard model remains to be fully investigated. But there is further evidence in favor of the above hypothesis in the fact that a polyvector field $\Psi=\psi^{\tilde{A}} \xi_{\tilde{A}}$ has 16 complex components. Altogether it has 32 real components. This number matches, for one generation, the number of independent states for spin, weak isospin and color (together with the corresponding antiparticle states) in the standard model. A complex polyvector field $\Psi$ has thus enough degrees of freedom to form a representation of the group $\mathrm{GL}(4, \mathrm{C}) \times \mathrm{GL}(4, \mathrm{C})$ which contains the Lorentz group $\mathrm{SL}(2, \mathrm{C})$ and the group of the standard model $\mathrm{U}(1) \times \mathrm{SU}(2) \times \mathrm{SU}(3)$. The generators of the group are given by $\Sigma_{A B}$ defined in eq. (29).

In the special case of free fields, the $C$-space Dirac equation (22) decouples into the set of four independent generalized Dirac equations for four independent 4-component spinors, each living in a different minimal left ideal:

$$
i \gamma^{M} \partial_{M} \psi^{\alpha i}=0, \quad i=1,2,3,4
$$

or explicitly,

$$
i\left(\gamma^{\mathbf{o}} \partial_{\mathbf{o}}+\gamma^{\mu} \partial_{\mu}+\gamma^{\mu \nu} \partial_{\mu \nu}+\gamma^{\mu \nu \rho} \partial_{\mu \nu \rho}+\gamma^{\mu \nu \rho \sigma} \partial_{\mu \nu \rho \sigma}\right) \psi^{\alpha i}=0
$$

A particular solution is

$$
\psi^{\alpha i}=u^{\alpha i} \mathrm{e}^{i P_{M} X^{M}}
$$

${ }^{7}$ The objects are considered as matrices in the generalized spinor indices $\tilde{A}, \tilde{B}, \tilde{C}, \tilde{D}$.

${ }^{8}$ The group $\mathrm{GL}(4, \mathrm{C})$ is subjected to further restrictions resulting from the requirement that the transformations (30) should leave the quadratic form $\Psi^{\ddagger} * \Psi$ invariant. So we have $\psi^{\prime \ddagger} * \Psi^{\prime}=\left\langle\psi^{\prime \ddagger} \Psi^{\prime}\right\rangle_{S}=$ $\left\langle S^{\ddagger} \Psi^{\prime \ddagger} R^{\ddagger} R \Psi S\right\rangle_{S}=\left\langle\Psi^{\ddagger} \Psi\right\rangle_{S}=\Psi^{\ddagger} * \Psi$, provided that $R^{\ddagger} R=1$ and $S^{\ddagger} S=1$. Explicitly, the quadratic form reads $\Psi^{\ddagger} * \Psi=\psi^{* \tilde{A}} \psi^{\tilde{B}} z_{\tilde{A} \tilde{B}}$, where $z_{\tilde{A} \tilde{B}}=\xi_{\tilde{A}}^{\ddagger} * \xi_{\tilde{B}}$ is the spinor metric. 
where $u^{\alpha i}$ satisfies

$$
\left(\gamma^{\mathbf{o}} p_{\mathbf{o}}+\gamma^{\mu} p_{\mu}+\gamma^{\mu \nu} p_{\mu \nu}+\gamma^{\mu \nu \rho} p_{\mu \nu \rho}+\gamma^{\mu \nu \rho \sigma} p_{\mu \nu \rho \sigma}\right) u^{\alpha i}\left(p_{\mathbf{o}}, p_{\mu}, p_{\mu \nu}, p_{\mu \nu \rho}, p_{\mu \nu \rho \sigma}\right)=0
$$

A spinor $\psi^{\alpha i}$ incorporates, besides the linear momentum excitations, also the area and volume modes, determined by $p_{\mu \nu}, p_{\mu \nu \rho}, p_{\mu \nu \rho \sigma}$. Those extra modes take into account the extended nature of the object. For a nice description of this latter concept on an example of the quenched minisuperspace propagator for $p$-branes see ref. [25].

However, in the interactive case (i.e., in curved $C$-space), we have the set of coupled equations (28) in which there occurs the $C$-space spin connection $\Gamma_{M}$. Using eq. (23) we can calculate the curvature according to

$$
\left[\partial_{M}, \partial_{N}\right] \xi_{\tilde{A}}=R_{M N}{ }^{\tilde{B}} \xi_{\tilde{B}}
$$

where

$$
R_{M N}{ }_{\tilde{A}}^{\tilde{B}}=\partial_{M} \Gamma_{N}{ }_{\tilde{A}}^{\tilde{B}}-\partial_{N} \Gamma_{M}{ }_{\tilde{A}}^{\tilde{B}}+\Gamma_{M}{ }_{\tilde{C}}^{\tilde{B}} \Gamma_{N}{ }_{\tilde{A}}^{\tilde{C}}-\Gamma_{N}{ }_{\tilde{C}}^{\tilde{B}} \Gamma_{M}{ }_{\tilde{A}}^{\tilde{A}}
$$

This is the relation for the Yang-Mills field strength. From the curvature we can form the invariant expressions, for instance $R_{M N}{ }^{\tilde{A} \tilde{B}}\left(\gamma^{M^{\ddagger}} * \xi_{\tilde{A}}\right)\left(\gamma^{N^{\ddagger}} * \xi_{\tilde{B}}\right)$ and $R_{M N}{ }^{\tilde{A} \tilde{B}} R^{M N}{ }_{\tilde{A} \tilde{B}}$ which can be used in the action as the kinetic term for the fields $\Gamma_{M}{ }_{\tilde{B}}{ }$.

Using eq.(29) we can express the spin connection in terms of the generators ${ }^{9}$ :

$$
\Gamma_{N}=\frac{1}{4} \Omega^{A B}{ }_{N} \Sigma_{A B}=A_{N}{ }^{A} \gamma_{A}, \quad A_{N}{ }^{A}=\frac{1}{4} \Omega^{C D}{ }_{N} f_{C D}{ }^{A}
$$

Inserting (49) into (48) we obtain

$$
F_{M N}^{A}=\partial_{M} A_{N}{ }^{A}-\partial_{N} A_{M}{ }^{A}+A_{M}^{B} A_{N}{ }^{C} C_{B C}{ }^{A}
$$

where $C_{B C}{ }^{A}$ are the structure constants of the Clifford algebra: $\left[\gamma_{A}, \gamma_{B}\right]=C_{A B}{ }^{C} \gamma_{C}$.

The $C$-space Dirac equation (28) can be split according to

$$
\left[\gamma^{\mu}\left(\partial_{\mu}+\Gamma_{\mu}\right)+\gamma^{\bar{M}}\left(\partial_{\bar{M}}+\Gamma_{\bar{M}}\right)\right] \psi=0
$$

where $M=(\mu, \bar{M})$, and $\bar{M}$ assumes all the values except $M=\mu=0,1,2,3$.

From eq.(49) we read that the gauge field $\Gamma_{M}$ contains:

(i) The spin connection of the 4-dimensional gravity $\Gamma_{\mu}^{(4)}=\frac{1}{8} \Omega_{\mu}^{a b}\left[\gamma_{a}, \gamma_{b}\right]$.

\footnotetext{
${ }^{9}$ We now omit the indices $\tilde{A}, \tilde{B}$.
} 
(ii) The Yang-Mills fields $A_{\mu}{ }^{\bar{A}} \gamma_{\bar{A}}$, where we have split the local index according to $A=(a, \bar{A})$. For $\bar{A}=\mathbf{o}$ (i.e., for the scalar) the latter gauge field is just that of $\mathrm{U}(1)$ group.

(iii) The antisymmetric potentials $A_{\mu \nu}, A_{\mu \nu \rho}, A_{\mu \nu \rho \sigma}$, if we take indices $A=\mathbf{o}$ (scalar) and $M=\mu \nu, \mu \nu \rho, \mu \nu \rho \sigma$.

We see that the $C$-space spin connection contains all physically interesting fields, including the antisymmetric gauge fields which occur in string and brane theories.

A caution is in order here. From eq. (49) it appears that there are only 16 independent generators $\gamma_{A}$ in terms of which a gauge field is expressed. But inspecting the generators (29) we see that there are more than 16 different rotations in $C$-space. However, some of them, although being physically different transformations, turn out to be mathematically described by the same objects. For instance, the generators $\Sigma_{a b}=\frac{1}{2}\left[\gamma_{a}, \gamma_{b}\right]$ and $\Sigma_{\tilde{a} \tilde{b}}=\frac{1}{2}\left[\gamma_{5} \gamma_{a}, \gamma_{5} \gamma_{b}\right]=\frac{1}{2}\left[\gamma_{a}, \gamma_{b}\right]$ are equal, although the corresponding transformations, i.e., a rotation in the subspace $M_{4}$ and the rotation in the dual space $\tilde{M}_{4}$ are in principle independent. Similarly we have $\Sigma_{1 \tilde{1}}=\frac{1}{2}\left[\gamma_{1}, \gamma_{5} \gamma_{1}\right]=\gamma_{5}=\Sigma_{\text {oõ }}=1 \gamma_{5}$. Such degeneracy of the transformations is removed by the fact that the transformation can act on the spinor polyvector $\Psi$ either from the left or from the right (according to (301)).

Returning to the Dirac equation (51) we see that besides the part having essentially the same form as the ordinary Dirac equation in the presence of minimally coupled 4dimensional spin connection and Yang-Mills fields, there is also an extra term which can have the role of mass if $\psi$ is an eigenstate of the operator $\gamma^{\bar{M}}\left(\partial_{\bar{M}}+\Gamma_{\bar{M}}\right)$. Since the metric signature of $C$-space is [10] (8+,8-) and the signature of the "internal" space is $(7+, 5-)$, the mass is not necessarily of the order of the Planck mass; it can be small due to cancellations of the positive and negative contributions.

\section{Conclusion}

The theory that we pursue here ${ }^{10}$ seems to be a promising candidate for the unification of fundamental interactions. It does not require inclusion of extra dimensions. Instead, it employs the degrees of freedom incorporated in the 16-dimensional Clifford space of the 4-dimensional spacetime. A curved Clifford space provides an interesting realization of Kaluza-Klein theory without the necessity of enlarging the dimensionality of spacetime. Such a fresh approach to unification which takes into account the ideas from various fash-

\footnotetext{
${ }^{10}$ See also references [12, 13, 10, 14.
} 
ionable theories, e.g., Kaluza-Klein theory, Clifford algebra, string and brane theory (branes sampled by Clifford numbers), is in my opinion very promising and deserves further more detailed investigation.

\section{Acknowledgement}

This work was supported by the Ministry of High Educution, Science and Technology of Slovenia

\section{References}

[1] M.B. Green, J.H. Schwarz and E. Witten, Superstring theory (Cambridge University Press, Cambridge, 1987).

[2] W. Pezzaglia, "Physical Applications of a Generalized Geometric Calculus," [arXiv: gr-qc/9710027; "Dimensionally Democratic calculus and Principles of Polydimensional Physics," [arXiv: gr-qc/9912025]; "Classification of Multivector Theories and Modifications of the Postulates of Physics", [arXiv: gr-qc/9306006].

[3] W. M. . Pezzaglia and A. W. Differ, "A Clifford Dyadic superfield from bilateral interactions of geometric multispin Dirac theory," arXiv:gr-qc/9311015 W. M. . Pezzaglia, "Polydimensional Relativity, a Classical Generalization of the Automorphism Invariance Principle," in V.Dietrich et al. (eds.) Clifford Algebras and their Applications in Mathematical Physics, 305-317 (Kluwer Academic Publishers, 1998) arXiv:gr-qc/9608052; W. M. . Pezzaglia and J. J. Adams, "Should metric signature matter in Clifford algebra formulations of physical theories?," arXiv:gr-qc/9704048.

[4] C. Castro, Chaos, Solitons and Fractals 10 (1999) 295; Chaos, Solitons and Fractals 12 (2001) 1585; " The Search for the Origins of M Theory: Loop Quantum Mechanics, Loops/Strings and Bulk/Boundary Dualities " [arXiv: hep-th/9809102; C. Castro, Chaos, Solitons and Fractals 11 (2000) 1663; Foundations of Physics 30 (2000) 1301.

[5] M. Pavšič, Found. Phys. 31 (2001) 1185 arXiv:hep-th/0011216; M. Pavšič, NATO Sci. Ser. II 95 (2003) 165 arXiv:gr-qc/0210060.

[6] M.Pavšič, The Landscape of Theoretical Physics: A Global View; From Point Particle to the Brane World and Beyond, in Search of Unifying Principle (Kluwer Academic, Dordrecht 2001).

[7] M. Pavšič, Found. Phys. 33 (2003) 1277 arXiv:gr-qc/0211085.

[8] M. Riesz, in Dixieème Congrès Math. des Pays Scandinaves, Copenhagen, 1946 (Jul. Gjellerups Forlag, Copenhagen, 1947), pp. 123-148.

[9] S. Teitler, Supplemento al Nuovo Cimento III, 1 (1965) and references therein; Supplemento al Nuovo Cimento III, 15 (1965); Journal of Mathematical Physics 7, 1730 (1966); Journal of Mathematical Physics 7, 1739 (1966).

[10] M. Pavšič, "Clifford space as a generalization of spacetime: Prospects for unification in physics," arXiv:hep-th/0411053 
[11] M. Pavšič, "Clifford space as a generalization of spacetime: Prospects for QFT of Point Particles and Strings" (in preparation).

[12] C. Castro and M. Pavšič, Phys. Lett. B 539 (2002) 133 arXiv:hep-th/0110079.

[13] C. Castro and M. Pavšič, Int. J. Theor. Phys. 42 (2003) 1693 arXiv:hep-th/0203194.

[14] C. Castro , "The Extended Relativity Theory in Born-Clifford Phase Spaces with a Lower and Upper Length Scales and Clifford Group Geometric Unification", Preprint, CERN EXT2004-128; 30 Sep 2004.

[15] F.D. Smith, Jr, Intern. J. Theor. Phys. 24 (1985) 155; 25 (1985) 355; J.S.R. Chisholm and R.S. Farwell, J. Phys. A: Math. Gen. 20 (1987) 6561;33 (1999) 2805; 22 (1989) 1059; J.P. Crawford, J. Math. Phys. 35 (1994) 2701; J.S.R. Chisholm, J. Phys. A: Math. Gen. 35 (2002) 7359; Nuov. Cim. A 82 (1984) 145; 185; 210; G. Trayling and W.E. Baylis, Int. J. Mod. Phys. A 16, Suppl. 1C (2001) 900; J. Phys. A: Math. Gen. 34 (2001) 3309; G. Roepstorff, "A class of anomaly-free gauge theories," arXiv:hep-th/0005079 "Towards a unified theory of gauge and Yukawa interactions," arXiv:hep-ph/0006065 "Extra dimensions: Will their spinors play a role in the standard model?," arXiv:hep-th/0310092, F. D. Smith, "From sets to quarks: Deriving the standard model plus gravitation from simple operations on finite sets," arXiv:hep-ph/9708379.

[16] D. Hestenes, Space-Time Algebra (Gordon and Breach, New York, 1966); D. Hestenes and G. Sobcyk, Clifford Algebra to Geometric Calculus (D. Reidel, Dordrecht, 1984).

[17] P. Lounesto, Clifford Algebras and Spinors (Cambridge University Press, Cambridge, 2001).

[18] W. Baylis, Electrodynamics, A Modern Geometric Approach (Boston, Birkhauser, 1999).

[19] G. Trayling and W. Baylis, J.Phys. A 34, 3309 (2001) ; Int.J.Mod.Phys. A 16S1C, 909 (2001).

[20] B. Jancewicz, Multivectors and Clifford Algebra in Electrodynamics (World Scientific, Singapore 1989).

[21] A. Lasenby and C. Doran, Geometric Algebra for Physicists (Cambridge U. Press, Cambridge 2002).

[22] A.M.Moya, V.V Fernandez and W.A. Rodrigues, Int.J.Theor.Phys. 40 (2001) 2347-2378 [arXiv: math-ph/0302007]; "Multivector Functions of a Multivector Variable" [arXiv: math.GM/0212223]; Multivector Functionals [arXiv: math.GM/0212224; W.A. Rodrigues, Jr, J. Vaz, Jr, Adv. Appl. Clifford Algebras 7 (1997) 457-466; E.C de Oliveira and W.A. Rodrigues, Jr, Ann. der Physik 7 ( 1998 ) 654-659. Phys. Lett bf A 291 (2001) 367-370. W.A. Rodrigues, Jr, J.Y.Lu, Foundations of Physics 27 (1997) 435-508.

[23] N. S. Mankoč Borštnik and H. B. Nielsen, J. Math. Phys. 43 (2002) 5782 arXiv:hep-th/0111257; J. Math. Phys. 44 (2003) 4817 arXiv:hep-th/0303224.

[24] K.R. Greider, Phys. Rev. Lett. 44 (1980) 1718; Found. Phys. 14 (1984) 467.

[25] A. Aurilia, S. Ansoldi and E. Spallucci, Class. Quant. Grav. 19 (2002) 3207 arXiv:hep-th/0205028; see also S. Ansoldi, A. Aurilia, C. Castro and E. Spallucci, Phys. Rev. D 64, 026003 (2001) arXiv:hep-th/0105027. 